\title{
Resolving gravitational microlensing events with long-baseline optical interferometry
}

\section{Prospects for the ESO Very Large Telescope Interferometer}

\author{
F. Delplancke ${ }^{1}$, K. M. Górski ${ }^{1,2}$, and A. Richichi ${ }^{1}$ \\ 1 European Southern Observatory, Karl-Schwarzschildstr. 2, 85748 Garching bei München, Germany \\ 2 Warsaw University Observatory, Aleje Ujazdowskie 4, 00-478 Warsaw, Poland \\ Received 6 February 2001 / Accepted 29 May 2001
}

\begin{abstract}
Until now, the detailed interpretation of the observed microlensing events has suffered from the fact that the physical parameters of the phenomenon cannot be uniquely determined from the available astronomical measurements, i.e. the photometric lightcurves. The situation will change in the near-future with the availability of long-baseline, sensitive optical interferometers, which should be able to resolve the images of the lensed objects into their components. For this, it will be necessary to achieve a milliarcsecond resolution on sources with typical magnitudes $\mathrm{K} \gtrsim 12$. Indeed, brighter events have never been observed up to now by micro-lensing surveys. We discuss the possibilities opened by the use of long baseline interferometry in general, and in particular for one such facility, the ESO VLT Interferometer, which will attain the required performance. We discuss the expected accuracy and limiting magnitude of such measurements. On the basis of the database of the events detected by the OGLE experiment, we estimate the number of microlenses that could be available for measurements by the VLTI. We find that at least several tens of events could be observed each year. In conjunction with the photometric data, our ability to measure the angular separation between the microlensed images will enable a direct and unambiguous determination of both their masses and locations.
\end{abstract}

Key words. gravitation - instrumentation: interferometers - techniques: interferometric - gravitational lensing

\section{Introduction}

The phenomenon of gravitational lensing, or multiple splitting of the image of a distant source in the gravitational field of an intervening object (a star, a galaxy, or a galaxy cluster), is perhaps one of the more spectacular demonstrations of the effects of general relativity. Gravitational lensing has been studied theoretically for many years (see e.g. Schneider et al. 1992), and has become an observed phenomenon with the discovery of the twin quasar Q0957+ 561 (Walsh et al. 1979). Many gravitational lensing systems have been discovered since, and the subject has grown into a very mature branch of astronomy.

In this paper we focus on the gravitational microlensing. This term, introduced by Paczyński (1986), describes gravitational lensing which can be detected only by measuring the intensity variation of a macroimage made of any number of unresolved microimages. An example of such an event occurs when a source star in the galactic bulge, or the LMC, is gravitationally lensed by the stars, or possibly other massive compact object, in our Galaxy.

Send offprint requests to: F. Delplanke,

e-mail: fdelplan@eso.org
Paczyński (1986) suggested that photometric surveys of $\sim 10^{6}$ stars in the Magellanic Clouds over time scales of $2 \mathrm{hrs}$ to 2 yrs should reveal, via microlensing effect, the massive, compact, dark objects in the Galactic halo.

Practical realisation of this proposal turned out to be possible just a few years later and several microlensing observing programs were conducted: MACHO (Alcock et al. 1993), EROS (Aubourg et al. 1993), OGLE (Udalski et al. 1992), and DUO (Alard et al. 1995). After providing the "proof of existence", that is the discovery of the gravitational microlensing in the Local Group, these programs aimed at the estimation of the lens population mass function, the optical depth to gravitational lensing in the Galaxy, and fundamental limits on the possible contribution to the total mass of the galactic halo from the massive, compact, and not directly observable, objects. Paczyński (1996) provides a comprehensive review of the subject and requisite theory of gravitational microlensing.

The splitting power of a compact lens of a mass of order of $1 M_{\odot}$ and located within a few kpc from us is only sufficient to separate the source images by about $\sim 1$ milliarcsec (mas), which is not resolvable by even the largest individual optical telescopes. However, in a relatively near future this situation will change. As we argue in this paper, 
the successful development of large optical interferometers, e.g. VLTI or Keck, will allow us, in conjunction with ongoing microlensing searches (which will provide prompt alerts) to follow the microlenses in real time, to spatially resolve them, to measure their image separations, and essentially to change the whole phenomenological framework of quantitative study of these events.

The main goal of this paper is to discuss the observational aspects of such studies, and to provide a statistical estimate of the performence of the method and of its applicability.

\section{Micro-lensing problem and interferometry}

The quantitative description of a simple single compact lens event depends on three physical parameters: the lens mass, $M$, its effective distance from us, $D$, and the relative angular velocity of the source and the lens, $V$. The effective distance is a combination of the lens and source distances, but in the Local Group microlensing observations the source distance is usually known. The photometric measurements of the individual lensing events cannot disentangle those three physical lens parameters. Indeed, typically only one constraint on those three parameters can be derived from the measured magnification history. In a few long duration events the auxiliary measurements were possible, since the effects of the motion of the Earth on the photometric variation of the images were discernible, and permitted to estimate the transverse velocity of the lens. This allows to derive two constraints on three parameters, and to reduce the parametric degeneracy of the lens model. In such rare cases the individual lens mass could be estimated as a function of the remaining unknown physical quantity, namely the exact distance to the lens. Interferometric measurements of the spatial extent of the lensing event, i.e. the angular separation of the images, or the amplitude of the light centroid wobble, will provide precious additional information with which unambiguous solutions for all lens parameters will be possible.

Two interferometric methods can be used to obtain the measurements of the angular scale of the microlensing event. One involves the high accuracy visibility measurements (described in Sect. 3.1), which determine directly the image separation. The other is the micro-arcsecond astrometry (see Sect. 3.2), which allows to measure the amplitude of the angular motion of the total image photocenter.

The photo-center of the image pair moves during the microlensing event and its location on the sky may wobble by up to a half of the lens-source angular separation. The amplitude of the photo-center wobbling depends strongly on the distance to the lens, and is maximised when the lens is located at mid-distance to the source. In this case, the amplitude of the wobbling can reach a few hundred micro-arcseconds. For example, with an image intensity ratio of 10 , and a maximum image angular separation of 1 mas, the wobbling amplitude would be of the order of $450 \mu$ arcsec.
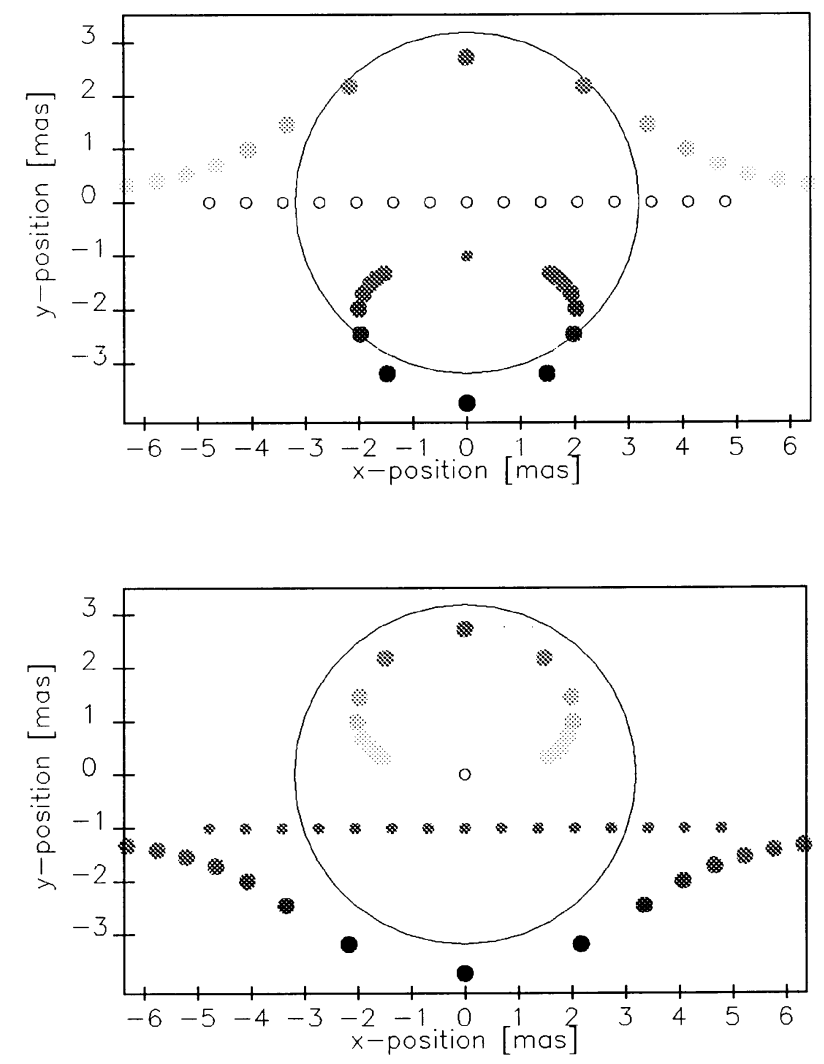

Fig. 1. Two representations of a microlensing event with $D_{\text {source }}=8 \mathrm{kpc}, M_{\text {lens }}=10 M_{\odot}, D_{\text {lens }}=4 \mathrm{kpc}$, and impact parameter of 1 mas. The radius of the Einstein ring for this event is $r_{\mathrm{E}}=3.2$ mas. Top panel - The background source position is fixed on the sky (small grey dot at $(x, y)=(0,-1)$ mas), and the lens is moving along the $x$ axis. Instantaneous pairs of lensed images are connected by the lines passing through the consecutive lens positions. Bottom panel - The lens position is fixed at the center of the frame, and the source position is shown moving along the line $y=-1$ mas.

A simple model of evolution of the configuration of microlensed images of a background star is shown in Fig. 1. The event is shown in two reference frames with either the fixed position of the background source and the lens moving in front of it (more natural for our discussion), or vice versa (usually preferred in microlening literature).

\section{Interferometric observations}

We consider a simple model of interferometric observations, which treats the microlensed image of a star as a compact double star, which cannot be resolved by individual optical telescopes (e.g. 1.8 or $8 \mathrm{~m}$ in the case of the VLTI). The elongation of each image due to the lensing effect is now assumed unresolved (but see Sect. 3.4 for further discussion of this point). The angular separation of the two images, of the order of a few milli-arcsec can be resolved by an interferometer as follows.

It is usually stated that an interferometer comprising two telescopes separated by a certain baseline $B$ has a resolving power of a single telescope of diameter $B$. But in 


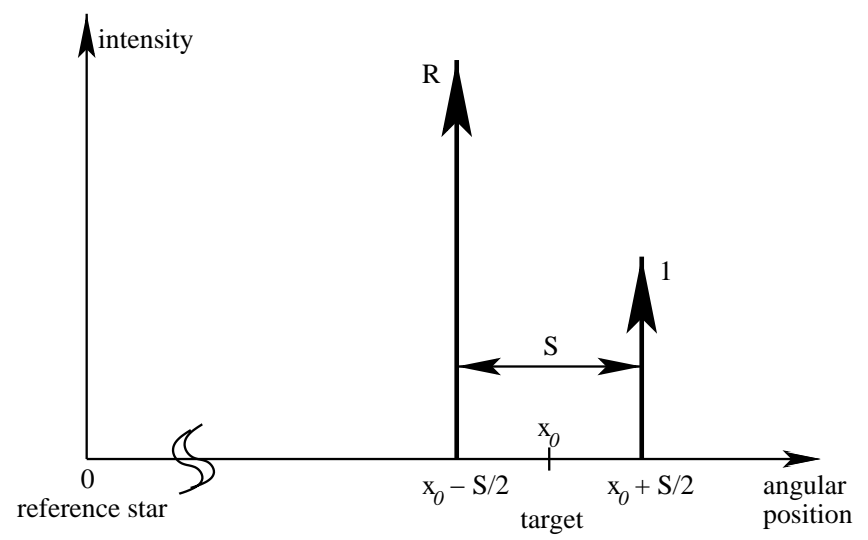

Fig. 2. A simple model of a double image of a gravitationally lensed star. The reference point can be arbitrary or can be the reference star when using the ESO VLTI PRIMA facility (Phase Referenced Imaging and Micro-arcsecond Astrometry).

practice a better performance can be achieved with observations of simple objects whose spatial structure can be easily modeled, like double stars, and interferometric observations can be used to effectively determine the model parameters (such as the star separation) beyond the simple resolution limit set by the baseline. This can be achieved either by accurate measurements of the fringe visibility, or by measuring the phase (i.e. the distance of the binary image photo-center to a reference star) with a very high accuracy. (This accuracy is expected to be at the level of $10 \mu$ arcsec in the astrometric mode of the VLTI.)

We proceed with discussion of both methods.

\subsection{Visibility measurements}

The microlensed image can be modeled (see Fig. 2) by two point sources, separated by a distance $S$ (projected on the interferometer baseline $B$ ), and with an intensity ratio $R$, which is related to the photometric amplification $A$ measured by the gravitational microlensing programs as follows:

$R=\frac{A+1}{A-1}$.

We shall limit our modeling to this 1-dimensional problem. The spatial function describing the model is given by:

$f(x)=R \delta\left(x_{0}-S / 2\right)+\delta\left(x_{0}+S / 2\right)$,

where $x_{0}$ is the distance from the reference star to the mid-point between the two images, and $x$ is the spatial coordinate (along the interferometer baseline).

The complex visibility measured by an interferometer, as a function of the interferometer baseline, $B$, is the Fourier transform of the spatial structure of the observed object (McAlister 1988):

$F(u)=R \mathrm{e}^{i 2 \pi u\left(x_{0}-S / 2\right)}+\mathrm{e}^{i 2 \pi u\left(x_{0}+S / 2\right)}$,

where $u=B / \lambda$, and $\lambda$ is the wavelength of observations.

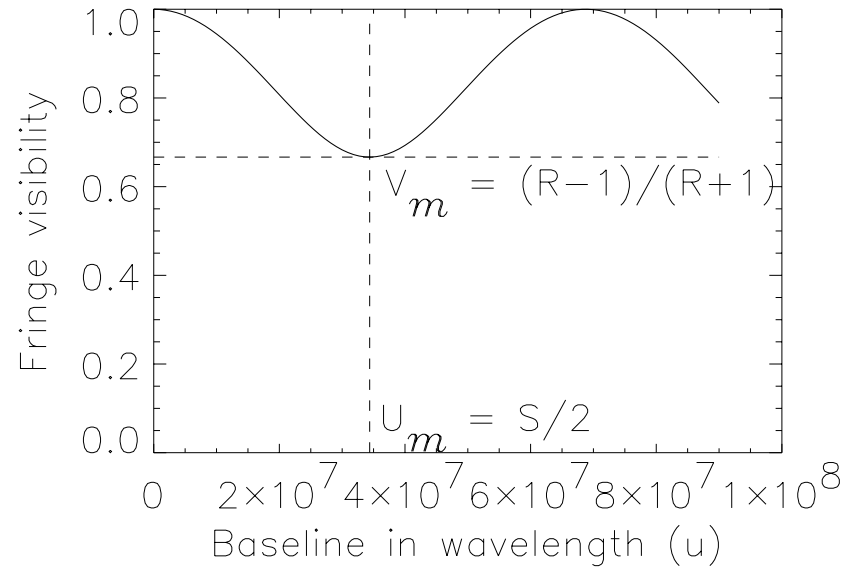

Fig. 3. Typical visibility curve as a function of the interferometer baseline (projected on the star splitting direction). The interferometer baseline is expressed in terms of observing wavelength.

The fringe scalar visibility, $V$, is the modulus of this Fourier transform, normalized by the total object intensity $(R+1)$. It is equal to:

$V(u)=\frac{|F(u)|}{R+1}=\frac{\sqrt{R^{2}+1+2 R \cos (2 \pi u S)}}{R+1}$.

This is a cosinusoidal function varying between $\frac{R-1}{R+1}$ and 1 (amplitude $\frac{2}{R+1}$ ) with a period $u=1 / S$ (see Fig. 3 ).

For small separations, barely resolvable by the interferometer $(u S \ll 1)$, the cosine function can be approximated by a parabola and the squared visibility is given by:

$V^{2}(u)=1-4 \pi^{2} \frac{R}{(R+1)^{2}} S^{2} u^{2}$.

From this equation, we can compute the absolute error on the star separation as a function of the measurement error on the visibility. Since the measurement errors on the intensity ratio are driven by photometric accuracy, and the baseline $B$ is measured accurately via the interferometric calibration, the error on the measured visibility is usually the dominant error source in the determined image separation. The absolute error on the separation (in radians) is then given by:

$\mathrm{d} S=\frac{(R+1)^{2}}{4 \pi^{2} R u^{2}} \frac{V}{S} \mathrm{~d} V$

Let us assume that an object with the image intensity ratio of $\sim 10$ is observed at wavelength $\lambda=2.2 \mu \mathrm{m}$ with a baseline of $130 \mathrm{~m}$, the maximum separation between the largest VLTI telescopes. If the accuracy of $\mathrm{d} V \sim 1 \%$ is achieved in the measurements of visibility, VLTI can detect a variation in the image separation as small as $\mathrm{d} S \sim 30 \mu \mathrm{arcsec}$, and this sensitivity improves with decreasing intensity ratio. The stated $1 \%$ accuracy of the measurement of visibility is expected with a good VLTI performance, to be achieved in observations of relatively bright objects - under very favourable circumstances this could be even up to a factor of 10 better. Later 
on we discuss this point more specifically in the context of expected performance of particular VLTI instruments.

With larger image separations only a few measurements of the visibility curve as a function of the baseline during a night are sufficient to constraint the simple double star model and to determine the image angular separation with the same accuracy. A typical interefrometric measurement has a cumulated observation time of $30 \mathrm{~min}$ on the star in order to average atmospheric effects; it is made of numerous shorter exposures ( 0.1 to typically $10 \mathrm{~s}$ with fringe tracking) and is accompanied by observations on calibration stars. Observation nights shall then be performed several times at intervals of days or weeks in order to obtain the time curve of the microlensing event.

Succesful measurements of the temporal evolution of the angular separation allow to determine the transverse velocity of the microlens. Thus, in principle, the measurement of the angular separation of the images and its time variation during the event, together with the estimate of the background source distance (obtained by other means), allow a complete determination of all microlense parameters, $M, D$ and $V$.

\subsection{Astrometric measurements}

Not only the image angular separation but also the photocenter location of the image pair can be measured by interferometry, using a special technique called narrow-angle astrometry. The astrometric observation method consists of the following. Two stars (a relatively bright reference one and the microlensed one), separated by up to some tens of arcsecond, are observed simultaneously with the interferometer. The configuration of the interferometer is such that fringes can be obtained simultaneously for both stars. Therefore, the interferometer must introduce a differential OPD (Optical Path Difference) between both star paths, linked intrinsically to the angular separation (projected on the baseline) between the stars. An internal metrology measures very accurately the differential OPD, or the angular separation of the stars (reference and target). With a $100 \mathrm{~m}$ baseline interferometer, a star separation of 10 arcsec, an integration time of $30 \mathrm{~min}$ (to average the perturbing atmospheric effects), and a metrology accurate at the $5 \mathrm{~nm}$ level, one can reach a distance accuracy measurement of $10 \mu \operatorname{arcsec}$ (Shao \& Colavita 1992a).

The astrometric measurements require specially designed interferometers like the PTI (Palomar Testbed Interferometer), the Keck-Interferometer, or the VLTI. The PTI currently reaches an accuracy better than $100 \mu$ arcsec, while the Keck-I and VLTI plan to reach the $10 \mu$ arcsec accuracy level. In this paper, we consider the VLTI equipped with PRIMA, as described in Sect. 4.

As with the visibility measurements, the measurement of the angular astrometric wobble of the lensed star image centroid as a function of time allows to solve independently for the lens mass and for the transverse velocity, provided the source distance is known.

\subsection{Numerical examples}

Here we present two examples of the photometric and visibility curves, and the astrometric photocenter wobble that could be measured during microlensing events specified as follows:

1. a source located in the galactic bulge (distance $8 \mathrm{kpc}$ ), lensed by a $10 M_{\odot}$ black hole, located at mid-distance, which passes by the line of sight to the source at 1 mas;

2. a source located in the Large Magellanic Cloud (distance $50 \mathrm{kpc}$ ), lensed by a $1 M_{\odot}$ halo object located at $10 \mathrm{kpc}$, which also passes by the line of sight to the source at 1 mas.

Both cases were studied over a range of separations between the lens and the source from 5 Einstein radia to the point of the closest approach. The microlensing event aspect and the interferometric results for case 1 and 2 are shown in Figs. 4 and 5 respectively.

These results show that both case 1 and case 2 can be studied with an interferometer performing either visibility measurements with a $1 \%$ accuracy, or astrometric measurements with a $10 \mu \operatorname{arcsec}$ accuracy.

We have also computed the lens mass limits for application of interferometric methods to study lenses in both Galactic Bulge and Large Magellanic Cloud. We kept the source and lens distances to the observer fixed and we considered a minimum angular separation between the lens and the source line of sight of 1 mas, as in the examples above. We considered separately measurements by visibility and by astrometry, with assumed accuracies of $1 \%$ and $10 \mu$ arcsec respectively. The minimum lens masses for which an interferometric signal can be detected are (visibility and astrometric limits in this order):

- in the Galactic Bulge case, $0.05 M_{\odot}$ and $0.01 M_{\odot}$;

- in the Large Magellanic Cloud case, $0.1 M_{\odot}$ and $0.05 M_{\odot}$.

It should be noted that, in all these cases, the detection limit is below the photometric detection threshold. The mass limit for a $1 \%$ photometric amplification is $0.1 M_{\odot}$ in both cases. The detection limit of photometric surveys is usually much larger than $1 \%$. There is no upper mass limit to the interferometric methods.

The limitations of interferometry for microlensing are linked to the magnitude of the lensed object and to the availability of an array with many different baselines. Until now optical interferometers did not reach sufficient sensitivity. Moreover, interferometric measurements on several baselines on the same night are needed to get either the visibility curve as a function of the baseline, or the 2-dimensional centroid wobble. Both limitations will be overcome by the generation of large interferometers currently under completion. 

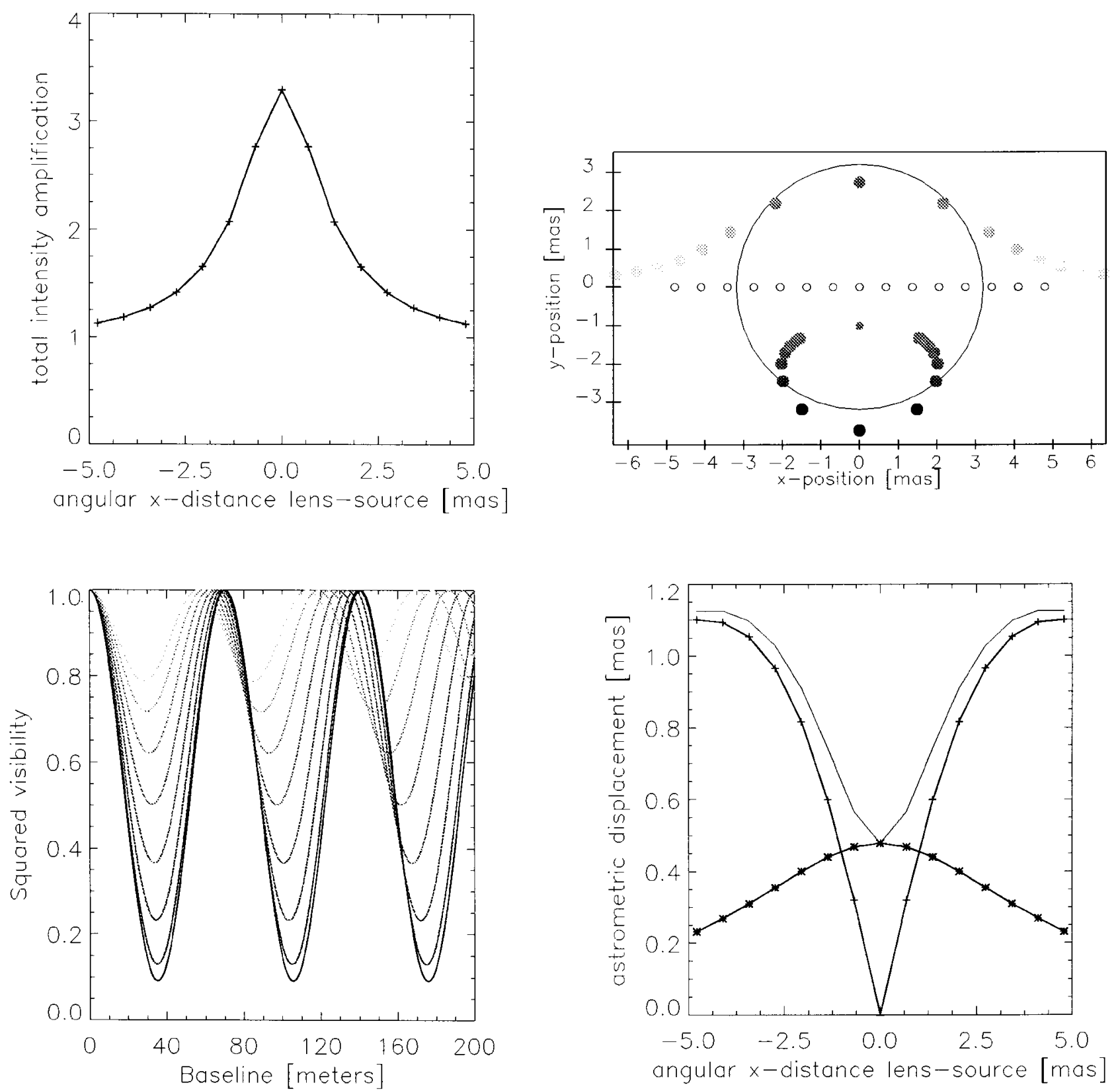

Fig. 4. Results of the simulation of the microlensing event with the following parameters: $D_{\text {source }}=8 \mathrm{kpc}, M_{\text {lens }}=10 M_{\odot}$, $D_{\text {lens }}=4 \mathrm{kpc}$, Einstein ring radius $r_{\mathrm{E}}=3.2$ mas, and the impact parameter of 1 mas. The microlensing event is sampled at discrete values of the lens-star angular separation as indicated on the photometric curve. Upper left: the photometric curve as a function of position of the lens. Upper right: the sky projection of the microlensing event showing the trajectory of the lens and the double images (the gray scale illustrates the image intensities), and the Einstein ring. Lower left: the visibility curves of the double-image for 8 points between the maximum $\left(5 r_{\mathrm{E}}\right)$ and minimum source-lens separation in the simulation. Lower right: the photo-center wobble as a function of time relative to the unlensed source position (crosses: along $x$-axis; stars: along $y$-axis; solid line: distance).

\subsection{Image elongation effect}

Depending on how close the lens is passing to the source line of sight and on the background source diameter, the 2 individual images of the source can be more or less elongated (see Fig. 6), and in the perfectly symmetric case of coaligned observer, lens, and source the lensed inmage is an Einstein ring. Hence, in principle, in some particular cases the image elongation could be resolved by an inter- ferometer and could influence significantly the visibility and astrometric measurements. To ensure the proper interpretation of the measurements the models should then be corrected accordingly.

As long as the arcs are not too long (no clear bending), the model correction consists in replacing the unresolved dots of the double star by two disks at the same separation. Both disks have the same diameter. So, just 

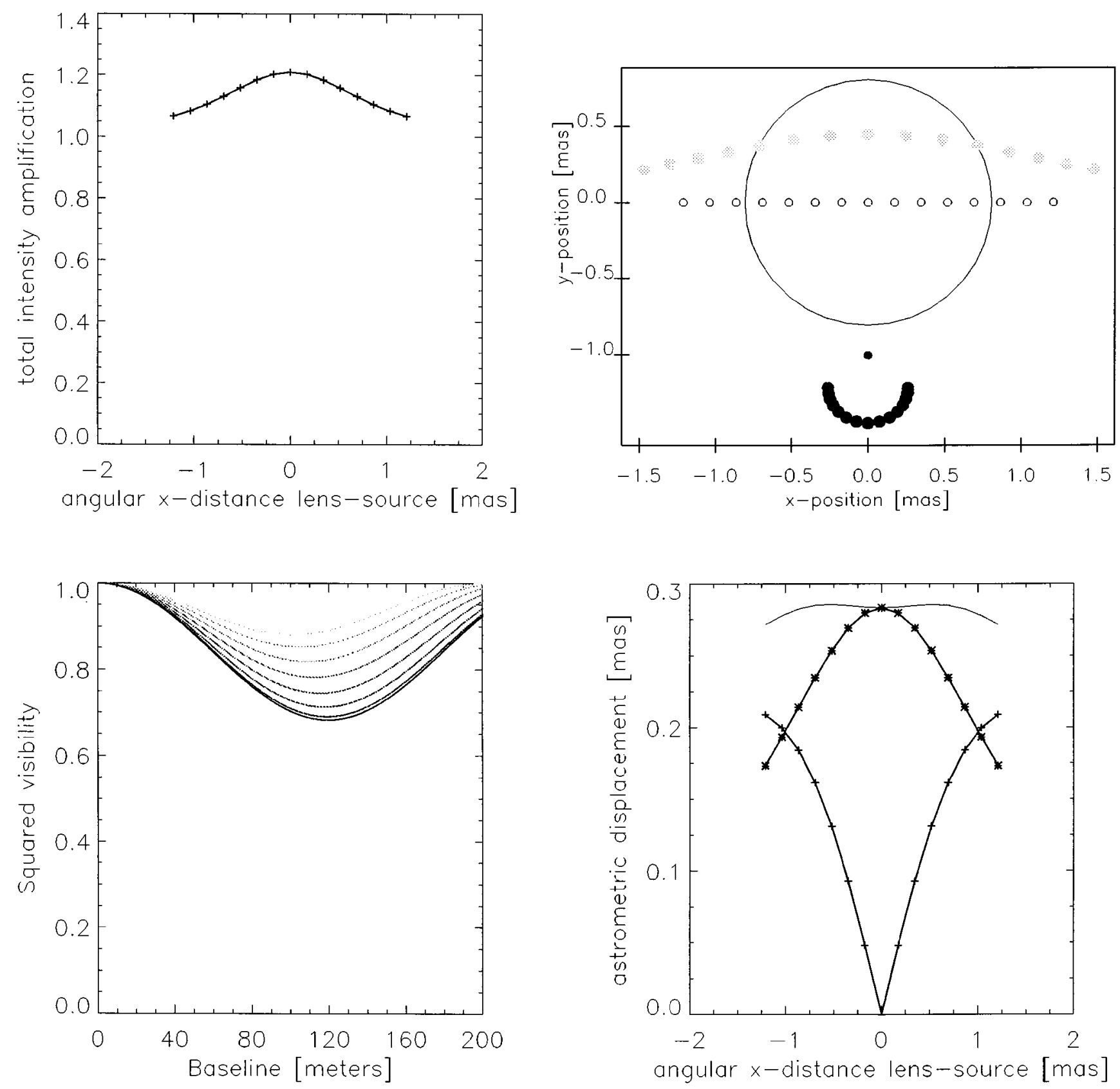

Fig. 5. Results of the simulation of the microlensing event with the following parameters: $D_{\text {source }}=50 \mathrm{kpc}, M_{\text {lens }}=1 M_{\odot}$, $D_{\text {lens }}=10 \mathrm{kpc}$, Einstein ring radius $r_{\mathrm{E}}=0.8 \mathrm{mas}$, and the impact parameter of 1 mas. Panel description is the same as for Fig. 4.

one parameter is added to the model. The cosine curve of Fig. 3 is modulated by a sinc function related to the disk diameter. This parameter can be easily solved for, by taking more measurements at different baselines. In this case, the astrometric measurement is not affected.

If the source-lens angular separation becomes small compared to the lens Einstein radius, the arc bending becomes significant and a 2-dimensional model of the microlensed image must be developed. The interpretation of the measurements is much more complex, and requires synthetic aperture imaging (Shao \& Colavita 1992b).

\section{Observing procedure of a microlensing event by an interferometer}

In this section, we provide some general guidelines for the observation of microlensing events with a generic longbaseline interferometer. A more detailed discussion in connection with the ESO VLTI facility is presented in Sect. 5 .

\subsection{Visibility measurements}

During one night several observations at different times are required for the projection of image separation on 


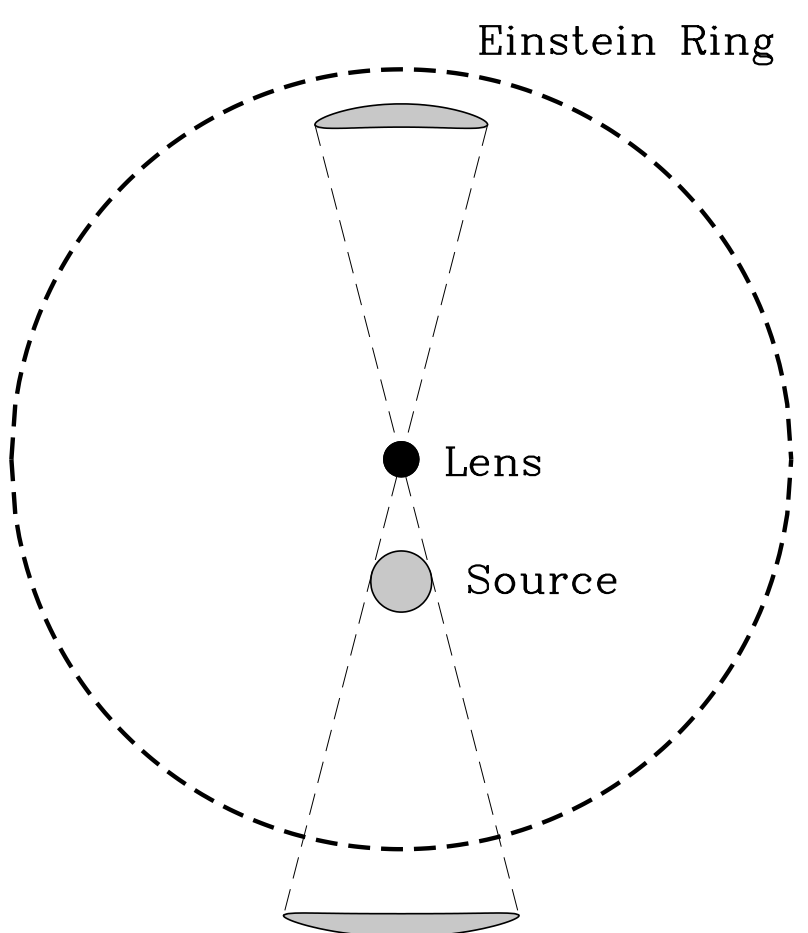

Fig. 6. Elongation of the two background source images for the microlensing event model (case 1) discussed in Sect. 3.3, and also shown in Fig. 4. The unlensed source diameter is $500 \mu$ arcsec. The image elongation is shown at the moment of the smallest lens-source separation, and the largest magnification during the event.

the interferometer baseline to vary significantly. Indeed, one measurement at a given time renders only one point on the visibility curve, and at least 2 points, at 2 different baselines are needed to constrain the curve given by equation 4 (2 parameters). The more points are observed, the more precise will be the separation measurement as the measurement error is random, and can be reduced by statistical methods. Depending on the required visibility measurement accuracy, the measurement points should be distributed over the full baseline range. With a $1 \%$ accuracy, only 4 or 5 points distributed over a $40 \mathrm{~m}$ baseline should suffice for an accurate fit.

Moreover, what is really measured is the separation of the image projected on the interferometer baseline. To have the absolute separation on the sky, at least 2 baseline orientations relative to the sky must be employed.

The baseline coverage can be increased in 3 ways:

- by using different telescopes forming different baselines; this will be possible either by observing on subsequent nights with different telescope configurations, or by using more than two telescopes in the same night;

- by using the Earth rotation to let the projection of the baseline on the stellar separation vary slowly; a star can be observed for at most 8 hours per night - this represents a variation of the projected baseline between $1 / 3$ and $2 / 3$ of its absolute value; if the night observing time is reduced due to the low elevation of the object, the baseline range will also be reduced;
- by measuring the binary image at different wavelengths; indeed, the microlensing event is achromatic (the separation and intensity ratio does not depend on the wavelength) but the resolution of the interferometer depends on the wavelength; an observation made at $10 \mu \mathrm{m}$ with a baseline of $130 \mathrm{~m}$ corresponds to an equivalent observation at $2.2 \mu \mathrm{m}$ made with a $28.6 \mathrm{~m}$ baseline (assuming, of course, that the background star properties do not depend on the wavelength; as the source is usually far away and not resolved, a variation of the source size or surface brightness with the wavelength can be neglected in most cases; moreover, the sources are mostly late type stars whose size does not vary with the wavelength between 1 and $2.5 \mu \mathrm{m}$ ).

\subsection{Astrometric measurements}

With astrometric observations, only 2 measurements per observation night are needed but the corresponding baselines must ideally be perpendicular, or in pratice forming an angle larger than $30^{\circ}$, in order to measure both components of the photo-center wobble.

\subsection{Scheduling}

The interferometer will receive alerts from photometric survey programmes when a sufficiently bright microlensing event of interestingly long duration (several weeks or more) begins. In the days following the alert, the interferometer shall observe the lensed star several times during one night. The observation shall be repated at regular intervals throughout the duration of the event.

\section{VLTI capabilities}

In practice, there will be very few facilities capable of performing the measurements proposed in this paper, including the ESO VLTI, the Keck, and the CHARA interferometers. The Keck interferometer will have access to only 1 baseline at a time with its two $10 \mathrm{~m}$ mirrors. With the less sensitive outrigger $1.8 \mathrm{~m}$ telescopes two perpendicular baselines will be available simultaneously. The CHARA array (Center for High Angular Resolution Astronomy) will access simultaneously up to 6 telescopes giving multiple baselines up to $400 \mathrm{~m}$ long, but with a smaller telescope size $(1 \mathrm{~m})$, and correspondingly less sensitivity.

The ESO Very Large Telescope Interferometer (VLTI) is a unique instrument for the measurements of image separation in microlensing events. When completed, the VLTI will provide 6 baselines with the high-sensitivity $8 \mathrm{~m}$ telescopes, and hundreds of baselines with the moveable $1.8 \mathrm{~m}$ auxiliary telescopes (several of which will be available during the same night). Science programs on the VLTI cover a very wide range, with no constraint to devote time to a specific key project. On the auxiliary telescopes, $100 \%$ of the time will be available for interferometry. Finally, the southern location of the VLTI is ideal for observing targets 


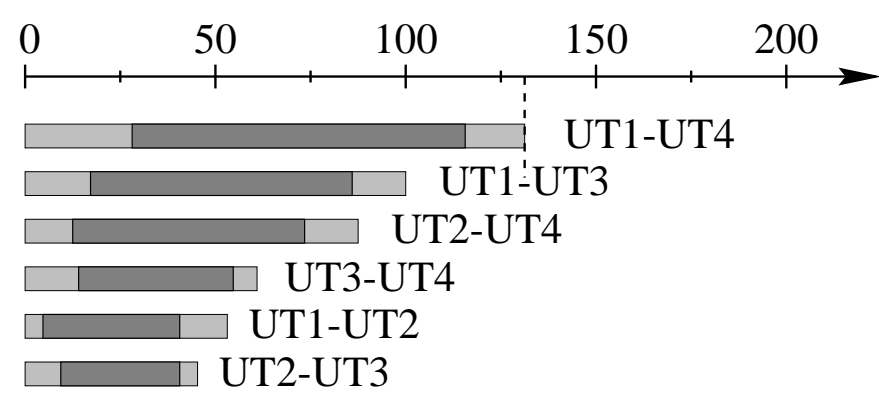

Fig. 7. Baseline coverage attainable with the various VLTI configurations, depending on the orientation of the image pair on the sky. The total baseline coverage is in light grey; the coverage attainable during 8 hours of observation is in dark gray, and amounts to $2 / 3$ of the light grey range and can slide on it.

in both the Galactic Bulge and the Magellanic Clouds, where most microlensing surveys are aimed.

The VLTI is following a progressive implementation plan (Glindemann et al. 2000). First light with the $40 \mathrm{~cm}$ siderostats has been achieved in March 2001. Unfortunately, such small telescopes cannot detect any microlensing event. The baseline coverage and expected sensitivity which will be implemented and achieved in various steps are summarized in Fig. 7 and Table 1. The instruments already available or under construction include two near-IR and one mid-IR beam-combiners, namely VINCI, AMBER and MIDI. The mid-IR range is not ideal for the observations proposed in this paper, and, hence, we will omit MIDI from the following discussion.

The VLTI test instrument VINCI will soon (writing in mid-2001) be used on the $8 \mathrm{~m}$ Unit Telescopes (UT). Atmospheric turbulence will limit the useful aperture to $\lesssim 2 \mathrm{~m}$, until an adaptive optics (AO) system will be available. VINCI will be capable of measuring visibilities with an accuracy of $2 \%$. Its limiting magnitude, with a $1.8 \mathrm{~m}$ collecting area, will be 8.3 in $K$-band. If an accuracy of $5 \%$ is sufficient, the limiting magnitude increases up to $K=9.8$. This capacity will be available by the end of 2001. The available UT baselines will increase progressively from 1 to 6 . The Earth rotation increases the $u-v$ plane coverage so that, even with only one baseline, microlensing measurements can be performed under favourable circumstances and with a carefully designed observation strategy (although exceptionally bright event would have to occur).

In 2003, the first two $1.8 \mathrm{~m}$ Auxiliary Telescopes (AT) will be available, with the same limiting magnitude as that reached with the $\mathrm{v}$ ignetted UTs without adaptive optics. This will increase the number of available baselines, especially due to the mobility of the ATs on the VLTI array. The array versatility and the dedication of the ATs exclusively to interferometry will represent a potential boost for microlensing studies, allowing to make measurements at each time a sufficiently bright event is detected.
In the second half of 2002 AMBER will be installed and used, initially with the ATs, and later with UTs and AO. The availability of AMBER will render a limiting magnitude of about 10, with a visibility accuracy of $2 \%$.

In mid-2003, an AO system will be available for the UTs, so that the full aperture can be used in $K$-band with VINCI and AMBER. The corresponding limiting magnitudes will then reach: 12.3 for VINCI with visibility accuracy of $2 \%, 13.8$ for VINCI with visibility accuracy of $5 \%$, and 13.4 for AMBER with visibility accuracy of $2 \%$.

By the end of 2003, the PRIMA facility (Phase Referenced Imaging and Micro-arcsecond Astrometry) will be installed on the Unit and Auxiliary Telescopes. It will provide two decisive advantages for measuring microlensing events, namely the capability to perform astrometry, and a large increase in limiting magnitude. Indeed, PRIMA will use a bright nearby reference star to stabilize the fringes on the target star. Longer integration times can then be used with all instruments and the limiting magnitude will increase.

The reference star must be $K \leq 10$ mag with the ATs, and of $K \leq 13 \mathrm{mag}$ with the UTs. The science object limiting magnitude then increases to $K \approx 16-18$ with the ATs, and 19-21 with the UTs, depending on the angular separation between the reference and science star. These will be the limiting magnitudes for microlensing observations. However, for visibility measurements (on the science star), the accuracy will probably be slightly degraded by atmospheric effects like anisoplanaticity and seeing variation over time (Tango 1980). The expected visibility accuracy with PRIMA is about $5 \%$.

The expected performances of the VLTI in the next four years are summarized in Table 1.

\section{Probability of microlensing event observations by the VLTI}

As discussed in the previous sections, the characteristics of a microlensing event depend on a sufficiently large number of parameters, that an estimate of the probability of observation by an interferometer must necessarily rely on a statistical approach. We have then chosen to use a sufficiently large, self-consistent list of microlensing events, and investigated how many among them could have been observed by the VLTI under one or more configurations.

For this, we have used the OGLE database of microlensing event observations towards the Galactic Bulge (Udalski et al. 2000). This includes 214 events observed between 1997 and 1999, for which the authors provide coordinates, baseline $I$-band magnitude, crossing time and amplification factors. The source coordinates fall approximately between $-25^{\circ}$ and $-40^{\circ}$ in declination, and are therefore ideally located for observations by the VLTI. One should also note that OGLE experiment is currently undergoing the camera upgrade (to an $8000^{2}$ $0.25^{\prime \prime}$-pixel camera) and should begin in the summer of 2001 a new, more efficient monitoring program OGLE III (Udalski 2001, private communication). The expected rate 
Table 1. VLTI configuration schedule and predicted statistics of microlensing detections.

\begin{tabular}{|c|c|c|c|c|c|}
\hline \multirow[t]{2}{*}{ Date } & \multirow[t]{2}{*}{ VLTI configuration } & \multirow{2}{*}{$\begin{array}{c}\text { Limiting mag. } \\
K\end{array}$} & \multirow[t]{2}{*}{ Baselines } & \multicolumn{2}{|c|}{ Detections/year } \\
\hline & & & & all & best \\
\hline end-2001 & $2 \mathrm{UT}$ (tip-tilt) + VINCI & $8.3-9.8$ & 1 & 0 & 0 \\
\hline mid-2002 & 4 UT (tip-tilt) + VINCI & $8.3-9.8$ & 6 & 0 & 0 \\
\hline end-2002 & $\mathrm{UT}$ (tip-tilt) or AT + AMBER & $9.6-10.3$ & numerous & 0 & 0 \\
\hline \multirow[t]{2}{*}{ mid-2003 } & $\mathrm{UT}+\mathrm{AO}+\mathrm{VINCI}$ & $12.3-13.8$ & numerous & 7.7 & 3.0 \\
\hline & $\mathrm{UT}+\mathrm{AO}+\mathrm{AMBER}$ & $12.9-13.4$ & numerous & 4.7 & 5.0 \\
\hline \multirow[t]{5}{*}{ mid-2004 } & $\mathrm{PRIMA}+\mathrm{AT}+\mathrm{VINCI}$ & $13-15$ & numerous & 19.0 & 15.3 \\
\hline & $\mathrm{PRIMA}+\mathrm{UT}+\mathrm{VINCI}$ & $16-18$ & 6 & $>63.3$ & $>61.3$ \\
\hline & PRIMA + AT + AMBER & $16.4-17.0$ & numerous & 60.0 & 44.0 \\
\hline & PRIMA + UT + AMBER & $19.6-20.1$ & 6 & $>63.3$ & $>61.3$ \\
\hline & PRIMA + AT + astrometry & $18-19$ & numerous & $>63.3$ & $>61.3$ \\
\hline
\end{tabular}

of discovery of gravitational microlenses should grow to about 500 events per year, and our estimates for plausible number of VLTI targets could be adjusted accordingly.

The microlensing phenomenon is achromatic, however the brightness of the lensed source can vary substantially with wavelength depending on its spectral type. Since the database under consideration has only a scarce coverage in spectral bands other than $I$, we cannot infer directly the $K$-band magnitude of the listed microlensed sources. Instead, we have used the fact that most of the field stars are $K$ and $M$ giants, and chosen an average correction of $I-K=1.5 \mathrm{mag}$, which would be appropriate for spectral types near M0III.

We have assumed two VLTI configurations, one in which the light is combined from two $8 \mathrm{~m}$ telescopes (UT) and one in which the auxiliary $1.8 \mathrm{~m}$ telescopes are combined (AT). Additionally, we have further considered whether an AO system is present and operating, or not. In all cases, we have considered that a fringe tracker is present and operating.

The difference between the above configurations is of course in the limiting magnitude that can be achieved. We have assumed that a suitable source is always present to operate the fringe tracker. Considering that the limiting magnitude for the fringe tracker currently under development for the VLTI is around $H \approx 13$, this condition should always be satisfied in the direction of the galactic bulge at least in the case in which the dual-feed facility PRIMA is available. In this case, also the conditions for the operation of the AO system would be fulfilled. Under these circumstances, we can adopt the magnitude limits presented in Sect. 4.

The angular resolution is in principle set by the baseline, and we do not concern ourselves with it at this point.

The number of objects that could have been detected in principle by the VLTI is listed in Table 1 for the different configurations considered above. We have listed in two different columns the situations in which all events are considered, or only the best ones. In the first case, we have assumed a detection if the magnitude of the object, includ- ing $1 / 2$ of the amplification effect, is within the quoted sensitivity limit. Under the best cases, we have considered those events in which also the actual baseline magnitude (i.e., in the absence of amplification) is within the quoted sensitivity limit, and in addition the characteristic crossing time is longer than 10 days. We consider this to be the minimum, for an event to be detected and recognized by a microlensing survey, and to be subsequently observed by the VLTI with at least two different baselines. Therefore, this definition of best cases includes those microlensing events which could in principle be observed by the VLTI during a large part of their evolution.

Note that for the configurations with the highest sensitivity, the listed frequency of detections should be considered as a lower limit only. This is due to the sensitivity limit of the available database. The faintest microlensing events detected by OGLE had a magnitude $I \approx 19.5$ (without amplification). When corrected by the $I-K$ color, this would be 1-2 mag brighter than the limit of some configurations of the VLTI, which could then in principle observe more objects than revealed by OGLE.

At face value, our simple analysis implies that $\mathrm{AO}$ and/or the dual-feed facility are needed, and therefore practical observations could begin after 2003. However, the uncertainties in the assumptions (in particular for the $I-K$ correction) are such that it is not impossible that a suitable event could be observed even earlier. Indeed, a spectacular microlensing event occured recently: OGLE-2000-BUL-43 involved a bright source star at $I_{0}=13.54 \mathrm{mag}$, and had a very long time scale $t_{\mathrm{E}}=156$ days (Soszyński et al. 2001). If a similar event occurs in the near future it will be an ideal target for the VLTI already in the early stage of development of the facility.

\section{Conclusions}

We have presented and discussed the possibility of observing microlensing events by means of a long-baseline, high-sensitivity interferometer such as the VLTI. Such 
observations, which could be attempted in the course of 2002 and become efficient by 2003/2004 with the availability of adaptive optics and the dual-feed facility, should permit to disentangle the degeneracy of parameters which currently limits the possibility to determine directly the mass and distance of the gravitational lens.

In particular, interferometric observations should allow us to measure the actual separations of the lensed images, which is typically at the 1 milliarcsec level, and to monitor their motion during the evolution of the phenomenon, either relative to each other or possibly to a fixed reference source.

We have discussed a possible observational strategy and the foreseen performance of the VLTI with its various subsystems for this kind of studies. We have also compared such performance against a database of observed microlensing events obtained from the OGLE experiment. The conclusion is that a very significant number of microlensing events could be observed in principle by the VLTI. We stress the importance of prompt alerts, and of near-IR photometry of the microlensing events.

Acknowledgements. We are grateful to B. Paczyński for the original idea and continuous encouragement of this work. This research has made use of the OGLE database, available at http://sirius.astrouw.edu.pl/.

\section{References}

Alard, C., Guibert, J., Bienayme, O., et al. 1995a, The Messenger, 80, 31

Alcock, C., Allsman, R., Axelrod, T., et al. 1993, Nature, 365, 621

Aubourg, E., Bareyre, P., Bréhlin, S., et al. 1993, Nature, 365, 623

Glindemann, A., Coudè du Foresto, V., Delplancke, F., et al. 2000, Proc. SPIE, 4006, 2

McAlister, H. A., ed. 1988, High Resolution Imaging by Interferometry, ESO Conf. and Workshop Proc., 29, 3

Paczyński, B. 1986, ApJ, 301, 503

Paczyński, B. 1996, ARA\&A, 34, 419

Schneider, P., Ehlers, J., \& Falco, E. E. 1992, Gravitational Lenses (Springer-Verlag, Berlin)

Shao, M., \& Colavita, M. M. 1992a, A\&A, 262, 353

Shao, M., \& Colavita, M. M. 1992b, ARA\&A, 30, 457

Soszyński, I., Żebruń, K., Woźniak, P. R., et al. 2001 [astro-ph00/12144], ApJ, in press

Tango, W. J., \& Twiss, R. Q. 1980, Progress in Optics, XVII, 239

Udalski, A., Szymański, M., Kałużny, J., Kubiak, M., \& Mateo, M. 1992, Acta Astron., 42, 253

Udalski, A., Zebruń, K., Szymański, M., et al. 2000, Acta Astron., 50, 1

Walsh, D., Carswell, R., \& Weymann, R. 1979, Nature, 279, 381 